\title{
Multi-periodicity of the Be star $\eta$ Centauri from spectroscopic and photometric observations
}

\author{
E. Janot-Pacheco ${ }^{1}$, S. Jankov ${ }^{1}$, N.V. Leister ${ }^{1}$, A.M. Hubert ${ }^{2}$, and M. Floquet ${ }^{2}$ \\ 1 Instituto Astronômico e Geofísico, Universidade de São Paulo C.P. 3386, 01060-970, São Paulo, SP, Brazil \\ 2 Observatoire de Paris, Section d'Astrophysique de Meudon, URA 335 du CNRS/DASGAL, F-92195 Meudon Cedex, France
}

Received May 25; accepted December 1, 1998

\begin{abstract}
We present photometric data and 209 highresolution, high signal-to-noise ratio optical spectra of the Be star $\eta$ Centauri obtained in 1993 and 1995, respectively. Time series analysis of these and other data, performed using the CLEAN, CLEANEST and Fourier Doppler Imaging techniques, show the presence of multiperiodic variations. They are interpreted in terms of low and high order non-radial pulsation modes. A strong frequency of $1.29 \mathrm{c} / \mathrm{d}$ is detected in line profile and photometric variations. It is attributed to a mode with $l=2$. Other high amplitude signals present in spectroscopic data are $1.78 \mathrm{c} / \mathrm{d}, 3.82 \mathrm{c} / \mathrm{d}$ and $4.51 \mathrm{c} / \mathrm{d}$. A frequency of $1.48 \mathrm{c} / \mathrm{d}$ appearing in spectroscopic data is compatible with the $1.56 \mathrm{c} / \mathrm{d}$ periodicity largely dominant in observations taken prior to 1993. The corresponding "superperiods" for four of the frequencies are commensurate at the $8 \%$ level. The star showed period and non-radial pulsation degree variations in timescales as short as $\sim 1.5$ hour. The dominant mode apparently alternates between $l=2$ and $l=4$ every other day during the eight day time span. This could be a modulation linked to the superperiod or to the stellar rotation. The main periodicities detected in our analysis are compatible with theoretical unstable $g$ modes in SPB variables. Short time scale variability $(1-3 \mathrm{hr})$, typical of high-order $p$ modes is also systematically present.
\end{abstract}

Key words: stars: emission-line, Be - stars: oscillations - stars: individual $(\eta$ Cen)

\section{Introduction}

High-resolution, high signal-to-noise spectroscopic observations have shown that the presence of multiperiodic variability in photospheric lines is rather common among

Send offprint requests to: E. Janot-Pacheco hot stars of luminosity class V-III (Kambe et al. 1990; Floquet et al. 1992, 1996; Gies \& Kullavanijaya 1988). Spectroscopic observations of rapid rotators (e.g., Be stars) with sufficient temporal resolution have shown that the observed line profile variability $(l p v)$ is characterized by the presence of "moving bumps" travelling from blue to red across the line profile in timescales of $\sim$ hours. The prototype object showing this kind of behavior is the Be star $\zeta$ Ophiuchi. The phenomenon has also been observed in O stars and $\delta$ Scuti variables (Kennelly et al. 1992, hereafter KWM) among others. Non-radial pulsations (NRP) have been proposed as an explanation for the $l p v$ observed in hot stars (e.g., Smith 1977; Gies 1991 and references therein). NRP produce $l p v$ thanks to the combination of the Doppler displacement of stellar surface elements with their associated temperature variations due to the compression/expansion caused by the passage of the waves through the photosphere. In favorable cases these variations can also be detected photometrically. In rapid rotators, there is a one-to-one correlation between the points in the broaded line profile and the position of inhomogeneities on the stellar surface (the so-called "Doppler Imaging"). The additional velocity fields due to NRP produce then series of "bumps" that will move from blue to red across the line profile due to the stellar rotation and wave motion (Vogt \& Penrod 1983).

Be stars are rapid rotators of spectral type late $\mathrm{O}$ to early A and luminosity class V-III, showing a near infrared excess and Balmer emission lines imputed to an equatorially concentrated circumstellar envelope, produced by sporadic mass ejection episodes. The causes of the "abnormal" mass loss are as yet unknown. In spite of their high $V \sin i$, rapid rotation alone cannot explain ejection episodes as Be stars are not observed to rotate sufficiently near the break-up velocity. NRP could be the clue to the Be phenomenon, providing the surplus of perturbation that is needed to cause mass ejection. Thanks to recent theoretical models that explain the excitation of NRP in hot stars through opacity mechanisms (Dziembowski \& 
Pamyatnykh 1991, 1993; Gautschy \& Saio 1993), the idea that $l p v$ in B-Be stars can be caused by NRP has been reinforced. In fact, the presence of NRP in B-Be stars (the so-called "Slowly-Pulsating Variables" - SPB) is a logical issue in the sense that it bridges the gap along the main sequence between $\beta$ Cephei and $\delta$ Scuti pulsating variables (Dziembowski 1997). In this paper, we present the results of an extensive search for periodic oscillations in the $\zeta$ Oph-type Be star $\eta$ Cen. The observational material consists of spectroscopic and photometric observations conducted at Laboratório Nacional de Astrofísica (LNA) (Pico dos Dias, MG-Brazil). Frequency analysis has been performed by means of algorithms employing Fourier techniques. Photometric and spectroscopic data of Cuypers et al. (1989) and Štefl et al. (1995, hereafter SBHB) and photometric observations obtained with the Hipparcos satellite are also reconsidered.

$\eta$ Cen (HD 127972; HR 5440; SAO 225044; MWC 232) is a rapidly-rotating $\left(V \sin i \simeq 360 \mathrm{~km} \mathrm{~s}^{-1}\right)$, bright $(V \simeq 2.3)$ early-type $\mathrm{Be} /$ shell star. Cuypers et al. (1989) and Cuypers (1991) found triple-wave and doublewave photometric modulations with periods $1.92 \mathrm{~d}$ and $1.28 \mathrm{~d}$, respectively. Bumps moving across the HeI $667.8 \mathrm{~nm}$ profile detected by Leister et al. (1994) were attributed to a NRP mode with $|m|=l \simeq 15$ or $|m| \approx 6$, $l \approx 7$. Variability in both $l p v$ (visible) and UV flux with a timescale of $1.64 \mathrm{c} / \mathrm{d}$ was detected by Gies (1994). Štefl et al. (1995) found nearly sinusoidal brightness (Strömgren system) and radial velocity variations (SiIII $\lambda 455.26 \mathrm{~nm}$ line) with period $p=0.64 \mathrm{~d}(1.56 \mathrm{c} / \mathrm{d})$. Finally, the Hipparcos catalogue (ESA 1997) quotes for the star a photometric period of $1.28 \mathrm{~d}(\equiv 0.78 \mathrm{c} / \mathrm{d})$.

\section{Observations and reductions}

Our spectroscopic and photometric observations were performed at the $1.6 \mathrm{~m}(\mathrm{~B} \& \mathrm{C})$ and $60 \mathrm{~cm}$ (Zeiss) telescopes of LNA, respectively.

\subsection{Spectroscopic observations}

Spectroscopic observations were obtained during six nights of March 1995 (over a total time span of eight nights, see Table 1) with the coudé spectrograph of the $1.6 \mathrm{~m}$ telescope. A EMI CCD camera $(1152 \times 770$ pixels) was used. Spectra were taken with a grating of $1800 \mathrm{l} / \mathrm{mm}$ (first inverse order) centered on the He I $\lambda 667.8 \mathrm{~nm}$ line. This arrangement yields a spectral resolution of $R \simeq 60000$ and a wavelength range of $\simeq 89 \AA$. The signal-to-noise ratio was typically $\simeq 250$ for exposure times between 300 and $900 \mathrm{~s}$. The usual bias, flat field and Th-Ar comparison lamp spectra were taken during each night. Observations were reduced with IRAF $^{1}$ using standard techniques for two-dimensional CCD data.

\subsection{Photometric observations}

Ten nights of $b$ (Strömgren) observations of $\eta$ Cen were obtained in May, July and August 1993 (Table 1) with a two-channel photometer attached to the $60 \mathrm{~cm}$ Zeiss reflector of Laboratório Nacional de Astrofísica (LNA). The mean integration time was $\simeq 5 \mathrm{~s}$. Only the stellar channel of the photometer is refrigerated and equipped with a $b$ filter; the second channel is simply used to monitor a comparison star in white light. As we were only interested in searching for periodic variations with timescales $\sim 1$ hour, data reduction consisted in fitting a low order polynomial to the stellar and comparison light curves for each night, and in determining the ratio variable flux/comparison flux for each Be star measurement. Frequency analysis was performed for the time series formed by these ratios.

\section{Frequency analysis}

A power spectrum obtained through a simple Fourier transform of a data string contains not only "true" frequencies present in the physical phenomenon under examination, but also many aliases introduced by data sampling. Combination of aliases can produce strong spurious peaks, and it is a difficult task to disentangle them from the "true" frequencies.

In our search for multiperiodicity in the selected $\eta$ Cen data sets we applied the Fourier analysis and two methods that try to get rid of aliases due to data sampling. They are the CLEAN (Roberts et al. 1987) and the CLEANEST (Foster 1995) algorithms implemented in a SUN workstation by Emilio (1997). Both methods are based on modeling the observed data in Fourier space as a combination of trial trigonometric functions. CLEANEST is more powerful, because it uses more than one trial function simultaneously. In the case of our spectroscopic data, frequency analysis was performed on each of the individual time series formed by data at each resolution step across the He I $\lambda 667.8 \mathrm{~nm}$ line profile. The $l p v$ were also analysed with the Fourier-Doppler Imaging technique (FDI) (KWM). In that method, the temporal variations of the line profile series are transformed in both time and Doppler space; a two-dimensional Fourier representation of the variations is thus obtained, where the frequencies along the velocity axis can be identified with the degrees $l$

\footnotetext{
${ }^{1}$ IRAF is distributed by the National Optical Astronomy Observatories, which is operated by the Association of Universities for research in Astronomy (AURA), Inc., under cooperative agreement with the National Science Foundation.
} 
Table 1. Log of observations at LNA

\begin{tabular}{lrllr}
\hline $\begin{array}{l}\text { Spectroscopy } \\
\begin{array}{l}\text { Date } \\
(1995)\end{array}\end{array}$ & $\begin{array}{c}\text { HJD span(d) } \\
(2449780+)\end{array}$ & $\begin{array}{l}\text { Number of } \\
\text { spectra }\end{array}$ & $\begin{array}{l}\text { Photometry* } \\
\text { Date } \\
(1993)\end{array}$ & $\begin{array}{c}\text { HJD span(d) } \\
(2449134+)\end{array}$ \\
\hline $11 / 03$ & $8.56-8.72$ & 21 & $24 / 05$ & $0.34-0.65$ \\
$12 / 03$ & $9.57-9.73$ & 21 & $25 / 05$ & $1.52-1.66$ \\
$15 / 03$ & $12.53-12.72$ & 44 & $26 / 05$ & $2.49-2.56$ \\
$16 / 03$ & $13.53-13.70$ & 52 & $09 / 07$ & $46.40-46.48$ \\
$17 / 03$ & $14.52-14.72$ & 52 & $10 / 07$ & $47.33-47.53$ \\
$18 / 03$ & $15.53-15.72$ & 19 & $11 / 07$ & $48.30-48.40$ \\
& & & $12 / 07$ & $49.30-49.31$ \\
& & & $29 / 07$ & $66.29-66.42$ \\
& & & $27 / 08$ & $95.29-95.39$ \\
& & & $29 / 08$ & $97.31-97.37$ \\
\hline
\end{tabular}

*Strömgren $b$ filter.

rather than with the (apparent) azimuthal orders (Gough, private communication to Kennelly et al. 1997).

\subsection{Spectroscopic data}

We searched for periodicity in the $l p v$ of the spectra taken at LNA. The CLEAN, CLEANEST and FDI algorithms were applied to 385 time series formed at each line-profile resolution element inside the profile. CLEAN and CLEANEST were also applied to the time series of RV, EW and FWHM measurements. Periods longer than about the total duration of the observing run, $\sim 7.16 \mathrm{~d}$ $(\nu=0.14 \mathrm{c} / \mathrm{d}$, which is also the formal frequency resolution) cannot be detected. The same procedure was applied to the RV, EW and FWHM data of SBHB.

\subsubsection{Line-profile variability}

High order $l p v$ is quite conspicuous in $\eta$ Cen. Additional low-order $l p v$ is also strongly present, as it can be seen in the peculiar average stellar line profile (see Fig. 1).Variations in line depth at the $\sim 1-2 \%$ level in time scales of $\sim 5 \mathrm{~min}$ are also observed in the central region of the HeI $\lambda 667.8 \mathrm{~nm}$ line. They are probably due to intrinsic Be-type variations probably arising in the circumstellar disk, not linked to NRP.

The CLEAN algorithm was applied to each of the 385 residual time series formed by subtracting the global mean spectrum of the observing run from each individual spectrum, and following the procedure of Gies \& Kullavanijaya (1988). The gain was set at 0.8 . The main peaks were identified after a few iterations, and there were no significant changes afterwards; we thus limited to 10 the number of iterations. A CLEANed periodogram at each position across the line is shown in Fig. 1 with the global average profile in the lower panel. Frequencies separated
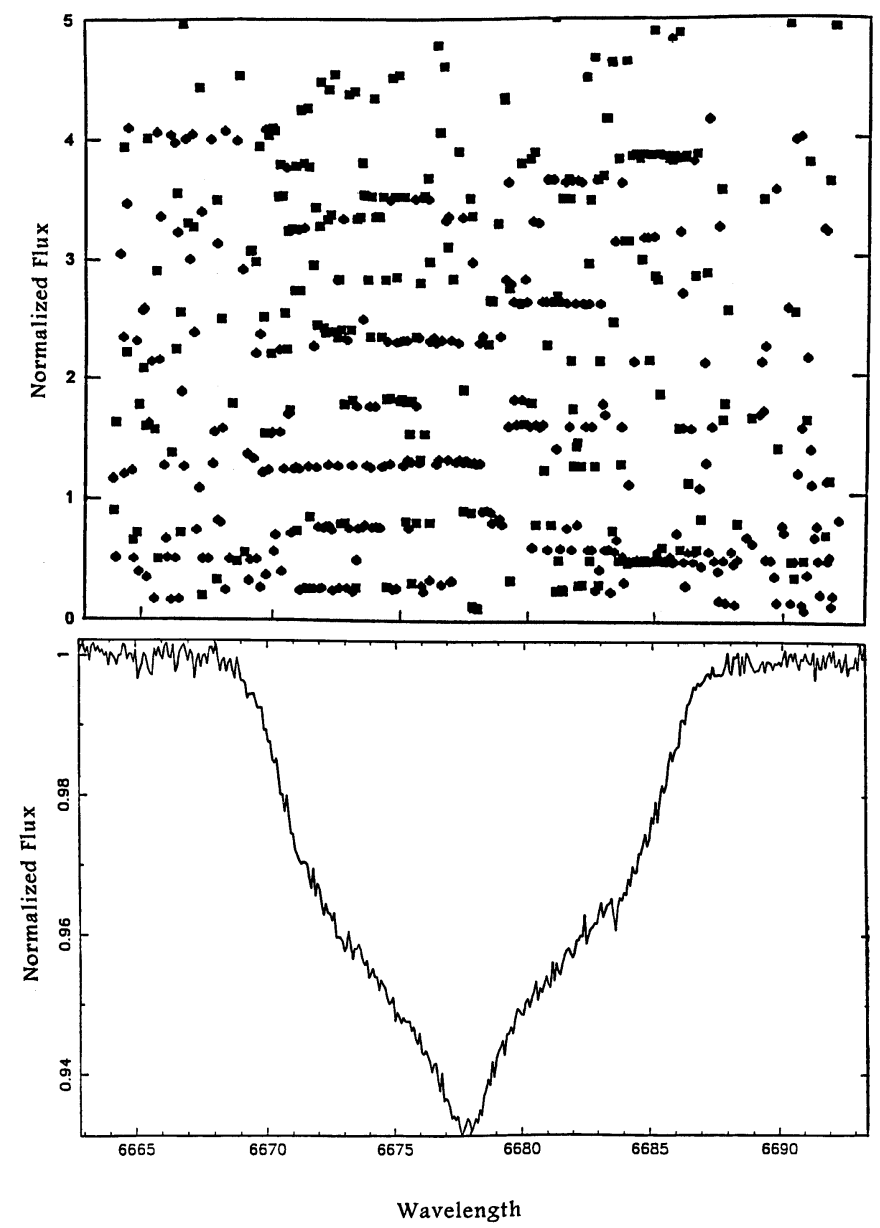

Fig. 1. Upper panel: global CLEANed periodogram at each position across the line profile. The first and second frequencies (by order of amplitude) found at each pixel are represented by a barred ellipse and a square, respectively. Lower panel: mean He I $667.8 \mathrm{~nm}$ line profile of $\eta$ Cen in 1995 March (Heliocentric wavelength) 
by $1 \mathrm{c} / \mathrm{d}$ can be combinations of a true frequency and an alias that CLEAN could not eliminate.

Indeed, the sequences $\sim 0.3 / 1.3 / 3.3$ and $1.7 / 2.7 / 3.7$ $\mathrm{c} / \mathrm{d}$ never occur together at any wavelength; only one of the frequencies of each group is probably present in the star.

We also applied the CLEANEST algorithm (Foster 1995) to our 385 time series across the line profile. This algorithm requires the number of frequencies to be defined a priori. We found that the clearest two-dimensional pattern is obtained by searching for just two frequencies. Since the method is applied to each of the 385 wavelength bin time series, more than two frequencies will show up (if more than two are present). In fact, we constructed two distinct residuals time series. A first one was formed by subtracting the global mean spectrum of the observing run from each individual spectrum; for the second one, we subtracted the mean nightly profile from each spectrum. For the first time series, lower frequencies are enhanced, while higher frequencies are more easily distinguished in the second series. Figure 2a shows the results of CLEANEST using the global mean spectrum; the corresponding periodogram summed across the wavelength axis is shown in Fig. 2b. The same is presented in Figs. 3a,b, but now using the nightly mean spectrum to form the residuals. Note that the algorithm was not able to eliminate completely the 1-day aliases.

The FDI method (KWM) was also applied to the 385 time series across the line profile. This technique maps the residuals onto a space where the coordinates are the time frequency and the normalized wavelength frequency, provided that the wavelength is mapped onto stellar longitude $\ell_{i}$ using:

$\ell_{i}=\sin ^{-1}\left(\frac{v_{i}}{v_{\mathrm{e}} \sin i}\right)$

where $v_{i}$ is the velocity corresponding to the wavelength bin $i$ with respect to the center of the line (KWM). It can be shown that the normalized wavelength frequency corresponds to the pulsation degree $l$. In the case of sectoral modes, it will naturally be equal to $l=|m|$. The main advantage of this technique is that it decomposes complex patterns of multiple modes. However, great care must be taken in the interpretation of the results, since simulations indicate that there is not a one-to-one relationship between an oscillation mode and its representation in Fourier space (e.g. Kennelly et al. 1997). The FDI was applied to the two residual time series as was done with CLEANEST. The projected rotational velocity is determined in Sect. 4.1. The variations of the line profile overall shape were decomposed by the FDI method into very strong low-degree pulsation modes $(l=2,4)$, although higher degree modes up to $l \sim 15$ can also be seen. In spite of the fact that the two-dimensional map is hampered by one-day aliases in the time frequency direction, the main frequencies coincide with that produced by CLEANEST.
Table 2. Frequencies (c/d) found in 1995 lpv using CLEANEST, by order of decreasing amplitude. Residuals have been obtained by doing a) spectra minus global mean spectrum, b) spectra minus nightly mean spectrum (all 6 nights together), and c) spectra minus nightly mean spectrum (each night analysed separately)

\begin{tabular}{llc}
\hline $\mathrm{a}$ & $\mathrm{b}$ & $\mathrm{c}$ \\
\hline 1.29 & 4.51 & 5.35 \\
1.78 & 3.82 & 7.80 \\
2.31 & 4.34 & 9.1 \\
2.78 & 3.67 & 10.5 \\
3.32 & 5.51 & 11.5 \\
1.48 & & 17.0 \\
0.76 & & \\
\hline
\end{tabular}

In order to examine the nightly variations, we also applied the CLEANEST and FDI methods to each night separately. In our case, the low limit time frequency that can be determined from each night is about $5.5 \mathrm{c} / \mathrm{d}$ (corresponding to $\sim 4.4 \mathrm{~h}$ nightly observing runs). The results are shown in Table 2 and Fig. 4 for nights May 15, 16. Note the changing pattern from night to night. In fact, for nights 11, 15 and 17 the two-dimensional map is apparently dominated by a mode with $l=\sim 2$, whereas for nights 12,16 and 18 a mode with $l=\sim 4$ is by far the strongest. However, one must be cautious about the quantitative aspect of this interpretation, because signals with frequencies $\leq 5.5 \mathrm{c} / \mathrm{d}$ are incompletely observed on a given night (see Sect. 4.3).

This apparent mode alternation will be discussed later (see the Discussion). For reasonable values of the stellar rotation frequency (see Sect. 4.1), high intensity modes lie generally above the non-propagating line, namely $\nu=$ $\nu_{\text {rot }}|m|$, which defines the position of surface phenomena that are locked to the stellar rotation. Those modes would thus correspond to prograde modes. Taking into account the extension in wavelength of occurrence of each frequency and from the power spectra summed across the wavelength axis (see Figs. $2 \mathrm{~b}$ and $3 \mathrm{~b}$ ), the main detected periodicities are summarized in Table 2. Other weak modes also appear at higher frequencies. For the moment, we will not discuss which frequencies are really present in the star; it appears clear, however, that even CLEANEST is not capable of eliminating all aliases due to data sampling. It is worth noting that the spectroscopic coverage is 1.07 days observed in total over a time span of 7.16 days. Clearly the large gaps in sampling cause significant ambiguities to exist in extracting the actual power spectrum from the data.

\subsubsection{Radial Velocity, FWHM and EW variations}

The CLEAN and CLEANEST algorithms have also been applied to the time series formed by the individual 
Apparent Time Frequency (cycles/day)
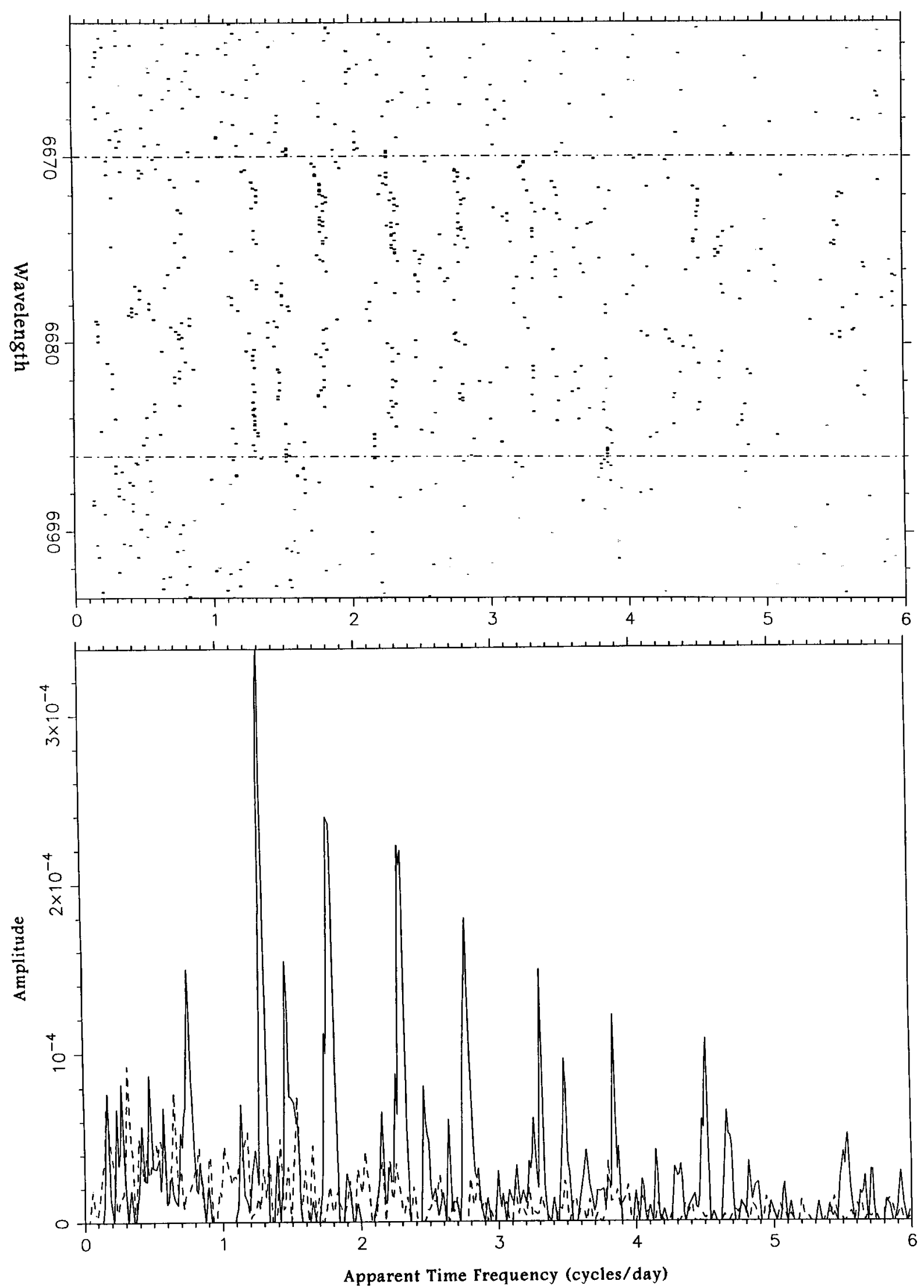

Fig. 2. a) Global CLEANESTed periodogram at each position across the line profile. The first and second frequencies (by order of amplitude) found at each pixel are plotted. Residuals were calculated with the global mean spectrum. The limits of the He I line are indicated. b) Corresponding periodogram summed across the wavelength axis inside (solid curve) and outside (dashed curve) the HeI line 
Apparent Time Frequency (cycles/day)
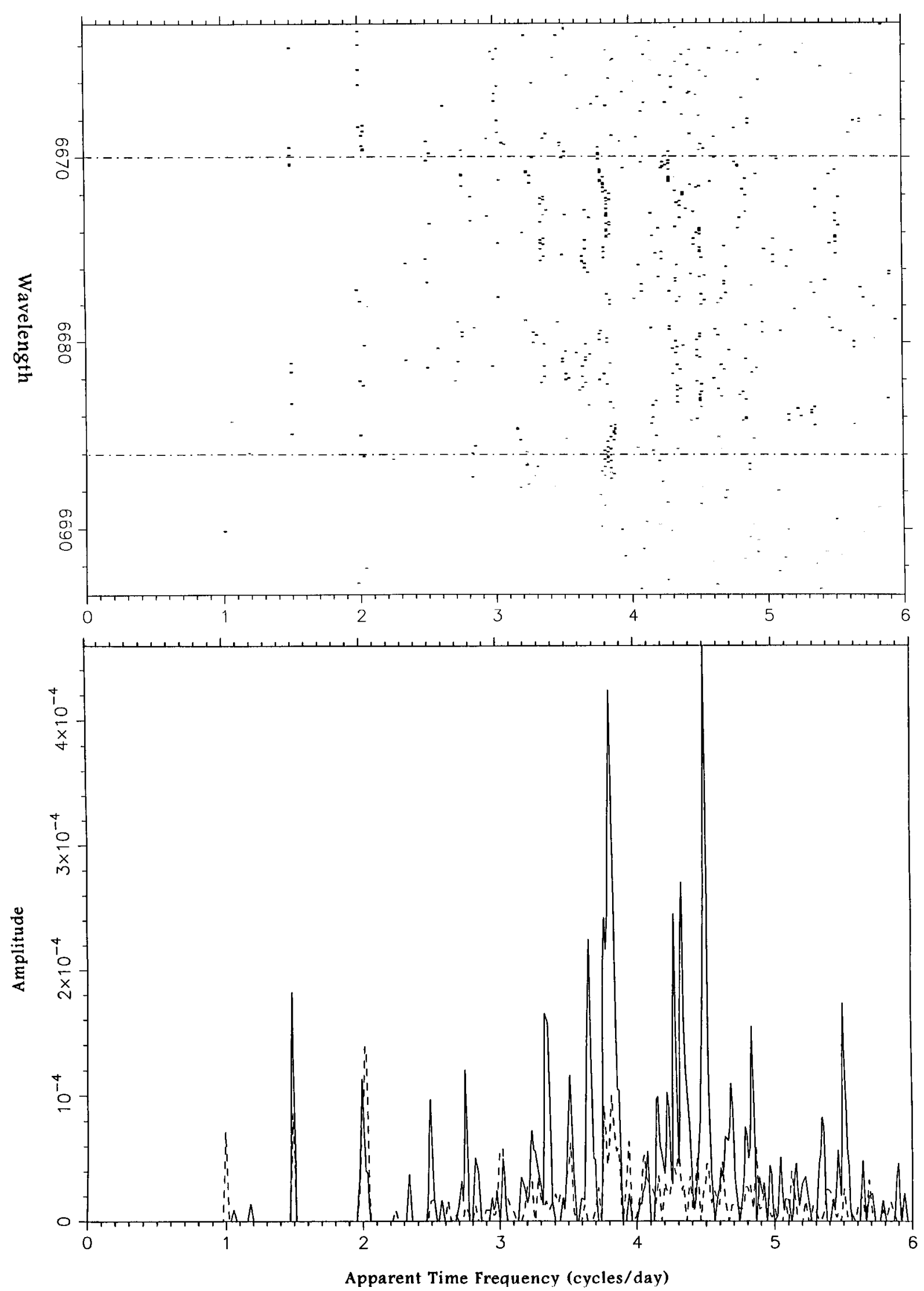

Fig. 3. Same as Fig. 2, but using the nightly mean spectra to construct the residuals 

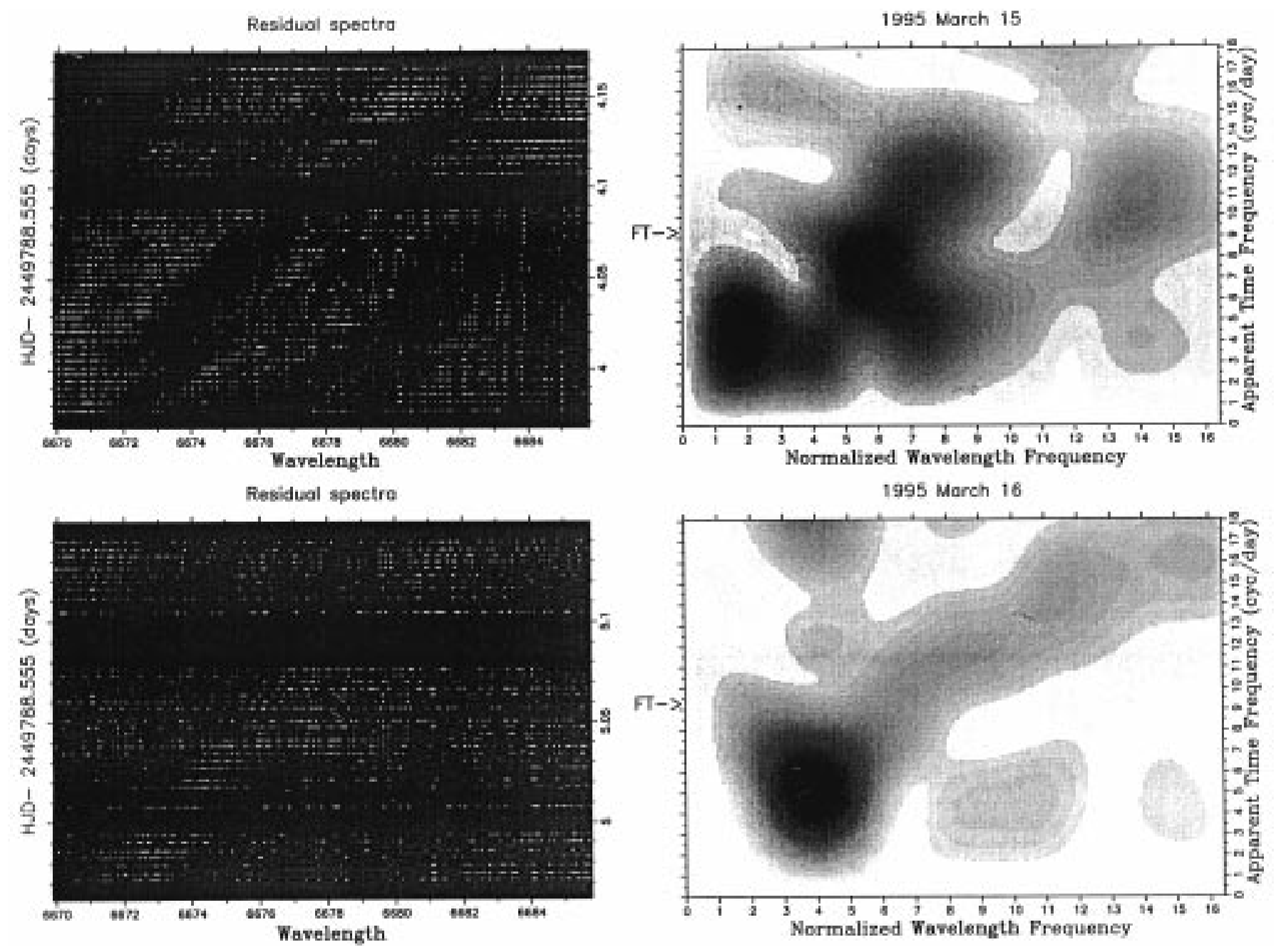

Fig. 4. a) left: Time evolution of the residuals from March 15. a) right: Fourier Doppler imaging of the corresponding line profile variations. The gray scale is normalized to the intensity of the highest $l \simeq 2$ peak. b) same as a) but for March 16 . Note the dominant $l \simeq 4$ mode

measurements of the radial velocity (RV), full width at half maximum (FWHM), and equivalent width (EW) of our HeI line observations. These parameters have been measured using standard IRAF procedures. Those two algorithms have also been used to analyse the temporal series of RV, FWHM and EW data measured by SBHB in Si III $\lambda 455.26 \mathrm{~nm}$ line profiles of $\eta$ Cen. The main frequencies found in these analyses are shown in Table 3.

\subsection{Photometric data}

We have searched for periodic variations in five different sets of photometric data (ground based using Strömgren $b$ filter): (1) data published by Cuypers et al. (1989) corresponding to observations made in 1987 and (2) in 1988, (3) Hipparcos data, obtained between early 1990 and August 1992, (4) data published by SBHB, corresponding to observations collected in 1992 and (5), observations obtained at LNA in 1993. The temporal analysis of these data sets was equally performed using both the CLEAN and CLEANEST algorithms, except for Hipparcos data which have been analysed with CLEAN. In Fig. 5 we show the dirty spectrum and the periodogram of LNA May-August 1993 data, obtained with CLEAN. The typical uncertitude on the frequencies detected in our photometric data is $\sim 0.08 \mathrm{c} / \mathrm{d}$, corresponding to the FWHM of the peaks in the periodogram. The main frequencies found in five data sets are:

(1) $1.36,2.45 \mathrm{c} / \mathrm{d}($ CLEAN) $1.29 \mathrm{c} / \mathrm{d}$ (CLEANEST);

(2) $1.56 \mathrm{c} / \mathrm{d}($ CLEAN) $, 0.56,1.59 \mathrm{c} / \mathrm{d}$ (CLEANEST);

(3) $1.56,2.10 \mathrm{c} / \mathrm{d}$ (CLEAN), 1.56, $2.13 \mathrm{c} / \mathrm{d}$ (CLEANEST);

(4) $1.56 \mathrm{c} / \mathrm{d}(\mathrm{CLEAN}), 1.56,3.23 \mathrm{c} / \mathrm{d}$ (CLEANEST);

(5) 1.56 (CLEAN).

These results compare well with time analysis of spectroscopic data (see above and Table 2). The $0.78 \mathrm{c} / \mathrm{d}$ quoted in the Hipparcos catalogue (ESA 1997) corresponds to a double-wave photometric curve; but in our frequency analysis the largely dominant periodicity is 
Table 3. RV, FWHM and EW variations

\begin{tabular}{|c|c|c|c|c|c|c|c|c|c|c|c|}
\hline $\begin{array}{l}\text { This paper }(\mathrm{He} \mathrm{I}) \\
\text { RV }\end{array}$ & & FWHM & & EW & & $\begin{array}{l}\text { SBHB (Si III) } \\
\text { RV }\end{array}$ & & FWHM & & EW & \\
\hline $\mathrm{C}^{*}$ & CL* & $\mathrm{C}$ & CL & $\mathrm{C}$ & CL & $\mathrm{C}$ & CL & $\mathrm{C}$ & CL & $\mathrm{C}$ & CL \\
\hline 0.76 & 1.28 & 1.27 & 1.78 & 0.62 & 0.67 & 1.55 & 1.64 & 2.45 & 2.45 & 0.50 & 0.68 \\
\hline 3.30 & 2.78 & 1.47 & 1.41 & 0.77 & 1.22 & 2.75 & & & & 2.18 & \\
\hline
\end{tabular}

${ }^{*} \mathrm{C}=\mathrm{CLEAN},{ }^{*} \mathrm{CL}=\mathrm{CLEANEST}$.
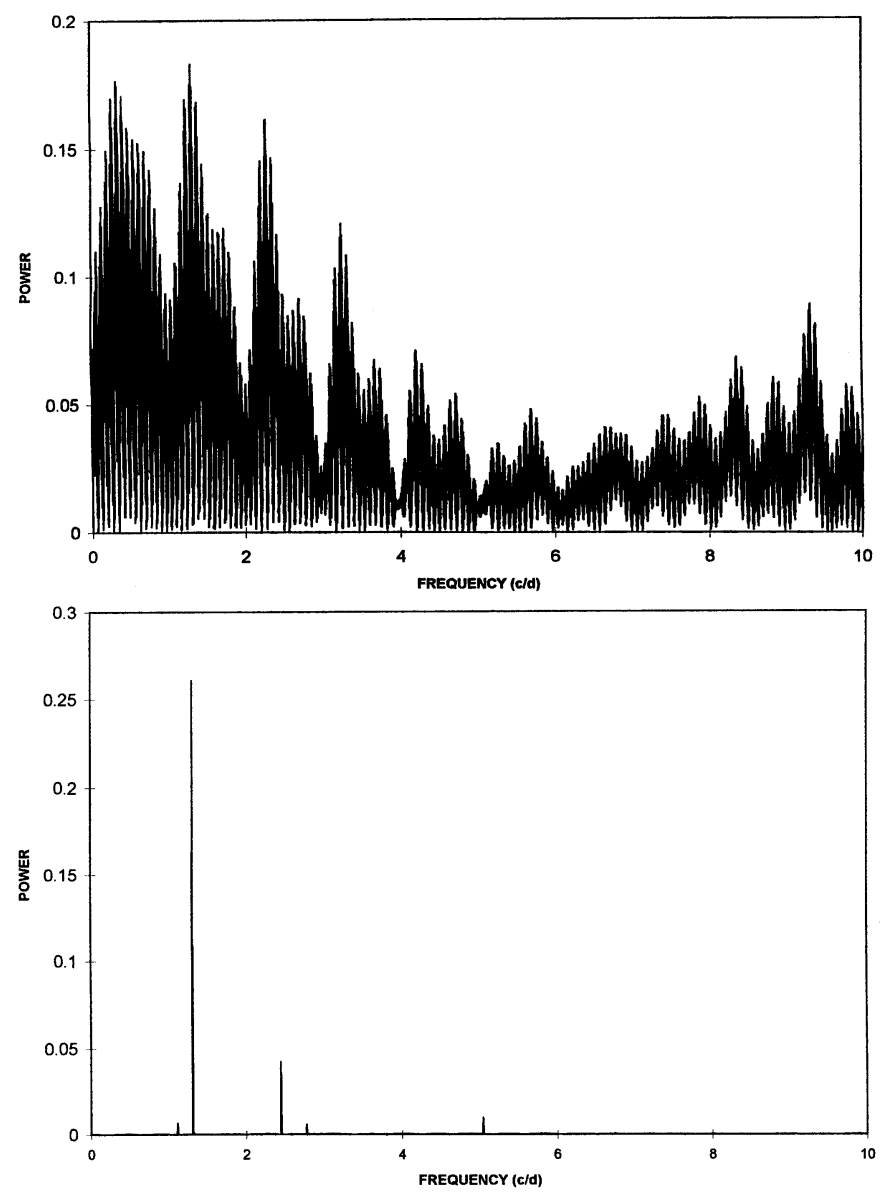

Fig. 5. a) Dirty spectrum for photometric measurements obtained at LNA (May-August 1993). b) Corresponding CLEANed periodogram

$1.56 \mathrm{c} / \mathrm{d}(=2 \times 0.78 \mathrm{c} / \mathrm{d})$, corresponding to a single-wave curve. It can be noted from the results of the frequency analysis of spectroscopic (RV, FWHM and EW) and photometric data, that the frequencies found with the two algorithms do not differ significantly. Moreover, allowing for precision and alias occurrence, some frequencies (e.g., $\sim 1.29,1.56 \mathrm{c} / \mathrm{d}$ ) are found in independent data sets. This strongly suggests that these frequencies most probably are not aliases due to data sampling.

\section{Discussion}

Our numerical simulations of time-series analysis indicates that CLEANEST is more efficient than CLEAN in identifying aliases due to the observational window (see Gies \& Kullavanijaya 1988, for a discussion about CLEAN on this matter). For that reason, in the following discussion more weight will be given to results obtained with CLEANEST but keeping in mind that this algorithm does not always succeed in eliminating aliases (see, for instance, Figs. 2, 3). Data sets obtained in five different epochs are analysed in this paper. We thus expect that power spectra will be essentially contaminated by one-day aliases (relatively easy to identify) and that periodicities appearing simultaneously in several data sets can reasonably be trusted to be present in the star. In the following, we will interpret the multiperiodicity detected in $\eta$ Centauri in the framework of NRP. Low-degree sectoral NRP modes $(l \approx 3)$ are detectable photometrically and in RV, EW and FWHM variations. They may also appear in lpv. In contrast, high-degree modes will not be detected photometrically due to cancellation effects. So, frequencies associated with low-degree modes can be identified by considering together the frequency spectra of the various kind of observable quantities.

\subsection{Stellar parameters}

The first minimum of the Fourier transformed mean spectrum can be used to estimate the projected stellar rotational velocity (Gray 1976). In the case of a Be star, factors such as gravitational darkening, shell components and shape deformation by the rapid stellar rotation can hamper the method and results must be treated with caution. Fourier transform has been applied to the mean spectrum derived from the six observing nights and from each night separately. While individual $\lambda 667.8 \mathrm{~nm}$ He I profiles show a considerable degree of asymmetry varying in time (that is, lpv) the mean profile is globally symmetric, indicating that positive and negative bumps corresponding to higher order NRP modes are averaged in time to its zero mean value (see Fig. 6, upper panel). Fourier frequencies are reduced to velocity units assuming that the first zero of the Fourier transform of a rotational profile points to the projected rotational velocity of the star 
(Fig. 6, lower panel). A limb darkening coefficient $\varepsilon=0.6$ was adopted. This analysis indicates a projected rotational velocity $V \sin i=350 \mathrm{~km} \mathrm{~s}^{-1}$ while the first minima of individual spectra indicate rather $360 \pm 30 \mathrm{~km} \mathrm{~s}^{-1}$, as can be seen for instance in Fig. 7. However, note that the second zero of the rotational mean profile is not at the expected position of about $200 \mathrm{~km} \mathrm{~s}^{-1}$, being biased towards $240 \mathrm{~km} \mathrm{~s}^{-1}$ (the third zero occurs at the expected position of $140 \mathrm{~km} \mathrm{~s}^{-1}$ ). This is certainly a consequence of the significant velocity fields introduced by other sources (as e.g., non-radial pulsations) present in the stellar atmosphere. Variations of several tens kilometers per second are sometimes observed in the Fourier transform minima from night to night. They are strong enough to smear the rotation pattern or to cause the first minimum to vanish. In the frame of the NRP model this behavior can be considered as a first indication of the presence of low degree $(l \approx 3) \mathrm{NRP}$ velocity fields that are superposed on the global rotation velocity field, deforming individual rotation profiles and producing the corresponding variations of the measured velocities. In what follows we will adopt $V \sin i=350 \mathrm{~km} \mathrm{~s}^{-1}$ as the stellar equatorial velocity.

The spectral classification of Be stars is a difficult task. Gravitational reddening caused by rapid rotation and variable influence of the changing, cooler and less dense envelope will often simulate a later spectral type and a higher luminosity class. The IAU CDS data bank (Strasbourg) lists spectral classifications in the range B1 V-B3 II. SBHB propose B1 III-V based on photometric data. Hipparcos measured for the star a parallax $\pi=10.57 \pm 0.39$ mas. Using $2.3 \leq V \leq 2.4$, $0.04 \leq E(B-V) \leq 0.06$ (quoted by SBHB) and $R=3.1$ we get $-2.84 \leq M_{V} \leq-2.56$. This puts an end to the controversy concerning the luminosity class of $\eta$ Cen: its absolute magnitude is typical of a B1-B2 main-sequence star, a giant class being formally excluded (e.g. Lang 1992). That classification range will be adopted hereafter.

The estimation of the rotational frequency and of the critical (break-up) depends on its mass and radius. Extreme values of these parameters for main-sequence B stars are given by Harmanec (1988) and Underhill (1982). The physical dimensions of a B1-2 V star are thus: $R=4.3-4.9 R_{\odot}, M=8.6-11.0 M_{\odot}$ (Harmanec) and $R=6.0-6.8 R_{\odot}, M=10.3-10.4 M_{\odot}$ (Underhill). In the Roche approximation, a star rotating at the critical (break-up) speed have $R_{\mathrm{e}}=1.5 R_{*}$. For the parameters quoted above, the extreme critical rotational frequencies (velocities) will be $\nu_{\text {crit }}=2.00 \mathrm{c} / \mathrm{d} \quad\left(V_{\text {crit }}=\right.$ $\left.744 \mathrm{~km} \mathrm{~s}^{-1}\right)$ and $\nu_{\text {crit }}=0.85 \mathrm{c} / \mathrm{d}\left(V_{\text {crit }}=440 \mathrm{~km} \mathrm{~s}^{-1}\right)$, respectively. Based in a line-fitting model, Hutchings et al. (1979) used UV observations to estimate independently rotation velocities and inclination angles for a sample of O-B stars. For $\eta$ Cen they obtain $i=55^{\circ}$ $\left(+12^{\circ},-7^{\circ}\right)$ and $V_{\text {rot }} / V_{\text {crit }}=0.65(+0.07,-0.12)$. Taking these values for granted, $V \sin i=350 \mathrm{~km} \mathrm{~s}^{-1}$, yields for $R_{\mathrm{e}}=R_{*}$ an equatorial velocity $V_{\mathrm{e}}=427 \mathrm{~km} \mathrm{~s}^{-1}(+44$,
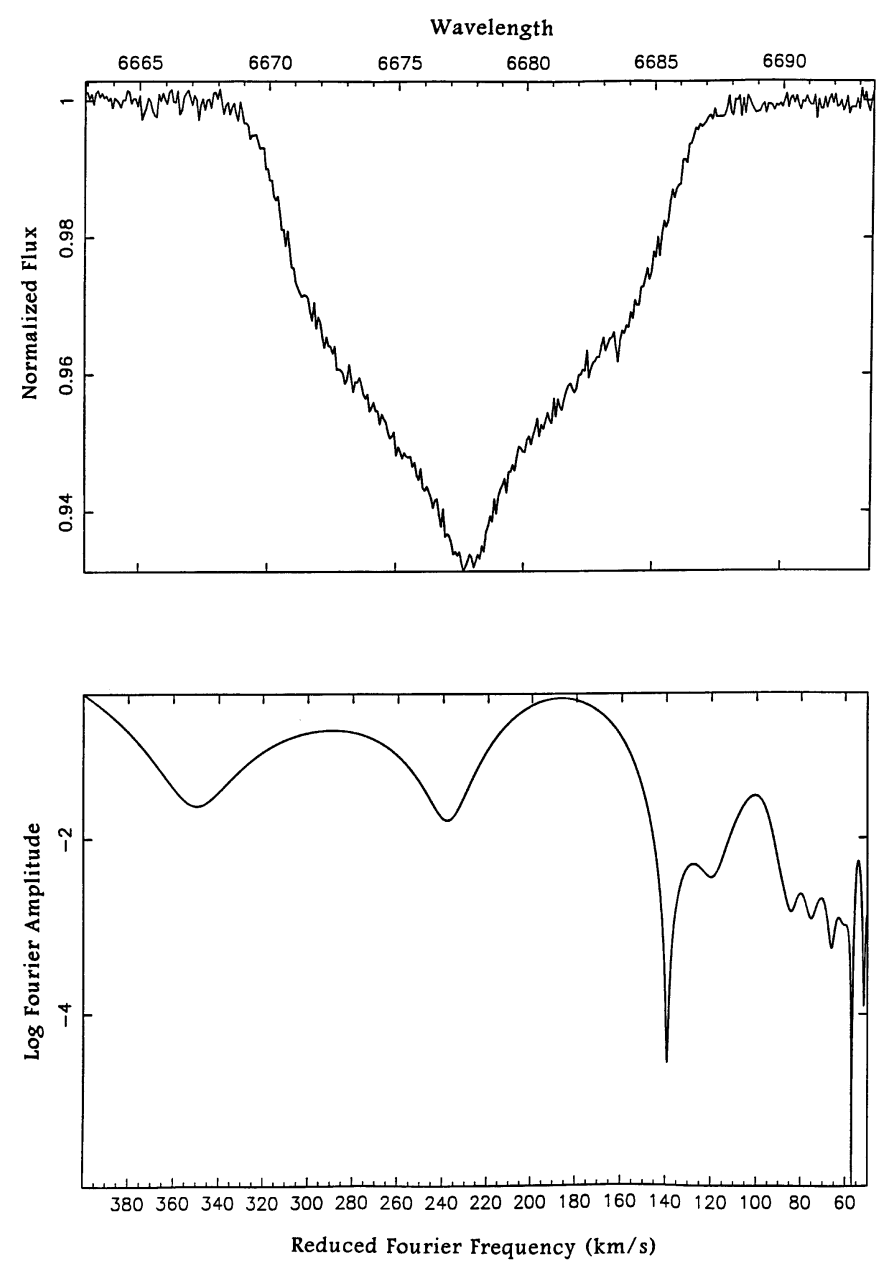

Fig. 6. Mean spectrum for the observing run (upper panel) and its Fourier transform (bottom panel)

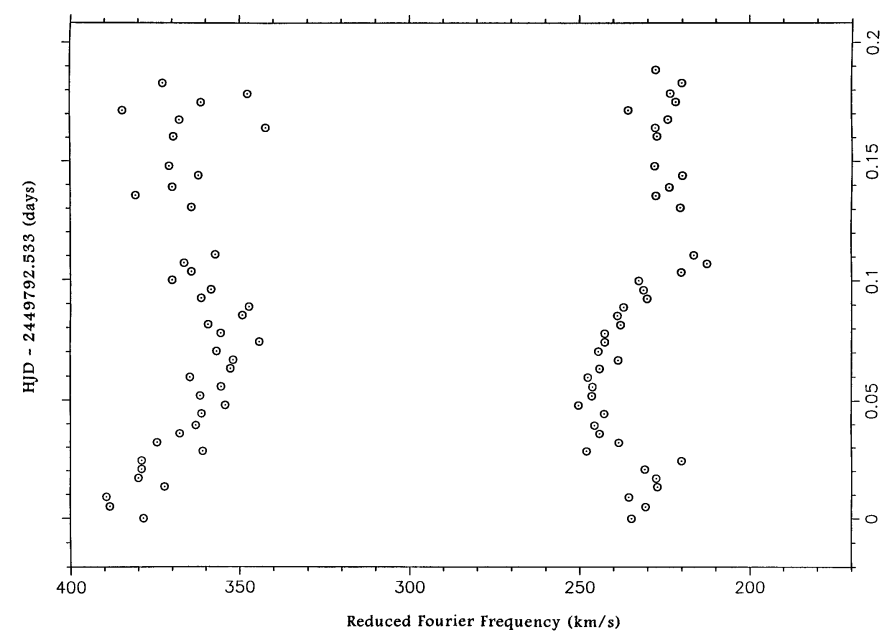

Fig. 7. Positions of the first and second minima of Fourier transformed line profiles for March 15 
$-47)$ and $1.10 \mathrm{c} / \mathrm{d} \lesssim \nu_{\text {rot }} \lesssim 2.16 \mathrm{c} / \mathrm{d} \quad\left(0.46 \mathrm{~d} \lesssim P_{\text {rot }} \lesssim\right.$ $0.91 \mathrm{~d})$. These results suggest that the mass and radius values of Harmanec (1988) are preferable in the present case. The critical frequency (period) of $\eta$ Cen is thus probably $\nu_{\text {crit }} \lesssim 2.28 \mathrm{c} / \mathrm{d}\left(P_{\text {crit }} \gtrsim 0.44 \mathrm{~d}\right)$. The definition of these parameters is important for the interpretation of the periodicities found in our time series analysis. For instance, frequencies greater than $\sim 2.3 \mathrm{c} / \mathrm{d}$ cannot be assigned to stellar rotation alone.

\subsection{NRP frequency and mode identification}

In the following, we will discuss the main frequencies detected in our temporal analysis (Table 2, Figs. 2, 3 and Sect. 3). Note that in the FDI analysis a first harmonic will have twice the $l$ value of the corresponding fundamental mode (cf. KWM). It should be kept in mind in the subsequent discussion that the frequency resolution for our photometric and spectroscopic data is $0.08 \mathrm{c} / \mathrm{d}$ and $0.14 \mathrm{c} / \mathrm{d}$, respectively. The reader should refer to Figs. 2 and 3 for $l p v$ results up to $6 \mathrm{c} / \mathrm{d}$.

1) The frequency $0.78 \mathrm{c} / \mathrm{d}$ is present in RV, EW and $l p v$ and can be partly due to a 1-day alias of $1.78 \mathrm{c} / \mathrm{d}$. It is mentioned in the Hipparcos catalogue, implying a doublewave light curve, but for a single-wave modulation the signal would correspond to a $1.56 \mathrm{c} / \mathrm{d}$ modulation (see Sect. 4.3).

2) The strongest modulation detected by CLEANEST in $l p v$ of our 1995 spectra when the global mean spectrum is used is $1.29 \mathrm{c} / \mathrm{d}$. It is also present in RV (CLEANEST) and FWHM (CLEAN) variations, and in our 1993 photometric data. The FDI analysis of all our spectroscopic data sets (hereafter FDIall for the sake of brevity) associates to it $l=1-3$. One and half-day aliases of this frequency $(2.3$ and $3.3 \mathrm{c} / \mathrm{d})$ appear in $l p v$ and other data sets. They never occur at the same time at any given wavelength.

3) A $1.56 \mathrm{c} / \mathrm{d}$ periodicity, stable and dominant in pratically all the data sets (photometric and spectroscopic, treated with both CLEAN and CLEANEST) obtained from 1987 through 1992 including Hipparcos data, if the variation is interpreted as a single-wave light curve. In this context, SBHB speculated that $1.56 \mathrm{c} / \mathrm{d}$ could be the stellar rotation frequency, the variability being due to the rotation modulation effect caused by one pattern of photospheric spots spanning the entire stellar circumference. This is in fact equivalent to a $l=|m|=1 \mathrm{NRP}$ pattern, whereas a double-wave variability corresponds to $l=|m|=2$ (cf. SBHB) (see below). The $1.56 \mathrm{c} / \mathrm{d}$ frequency is absent or very weak in LNA 1993 photometry, but it can be identified with a $1.48 \mathrm{c} / \mathrm{d}$ secondary peak appearing in our 1995 lpv and FWHM periodograms. The same could be said with respect to a $0.61 \mathrm{~d}$ modulation (三1.62 c/d) which was detected in a multiwavelength spectroscopic campaign conducted in 1991 March-April (Gies 1994). Beating and/or NRP amplitude variations could explain the fact that $1.56 \mathrm{c} / \mathrm{d}$ is by far the strongest periodicity present in $\eta$ Cen in 1987, 1988 and 1992, whereas it is fairly weak during 1993 and 1995 . We will return to the matter at the end of this discussion.

4) A $1.78 \mathrm{c} / \mathrm{d}$ periodicity is distinctly present in $l p v$ (CLEAN and CLEANEST) and is also weakly present in some photometric, EW and FWHM data sets. It could be 2-day alias of $1.29 \mathrm{c} / \mathrm{d}$. This periodicity has been recently reported by Štefl et al. (1997) as a secondary signal appearing in spectroscopic material obtained during 1995-1997. The one-day alias of this frequency $(2.78 \mathrm{c} / \mathrm{d})$ is clearly seen in our $l p v$ periodogram.

5) The frequency $3.82 \mathrm{c} / \mathrm{d}$ is quite conspicuous in the $l p v$ analysis performed with the nightly mean spectrum (CLEANEST, Fig. 3b). This frequency appears in FDIall associated with $l=1-3$, and the phase difference across the line profile indicates $l=1.9$.

6 ) A peak at $\sim 4.34 \mathrm{c} / \mathrm{d}$ appears in the periodogram of $l p v$ obtained with CLEANEST, using the nightly mean spectra. This frequency corresponds to $l=2-4$ in the FDIall results.

7) A variation with $4.51 \mathrm{c} / \mathrm{d}$ is the strongest in the $l p v$ analysis with CLEANEST and appears also in CLEAN results. FDIall map associates it to $l=3-5$. The $5.5 \mathrm{c} / \mathrm{d}$ frequency present in the $l p v$ periodogram (Fig. 3b) (presumably appearing also in Table 2, Col. c as $5.35 \mathrm{c} / \mathrm{d}$ ) is probably a one-day alias of this strong modulation.

8) The frequency $7.8 \mathrm{c} / \mathrm{d}$ is clearly present in the nightly analysis with CLEANEST and the FDI method, where it appears associated with $l=6$ (cf. Fig. 4a). This periodicity (or twice it) was detected by Leister et al. (1994), and tentatively associated with a tesseral $l \sim 7, m \sim 6$ mode.

9) Finally, periodicities of $\sim 9.1,10.5,11.5$ and $17.0 \mathrm{c} / \mathrm{d}$ are also clearly present with moderate to low amplitude in some of the nights analysed separately with CLEANEST and in FDI results (see Fig. 4). It must be remembered that higher frequencies show up more easily when the nightly mean spectrum is used for constructing the residuals. Further observations with higher $\mathrm{S} / \mathrm{N}$ are needed to establish the nature of such rapid periodicities, which would be characteristic of $p$ modes. This has also been observed in the B star $\iota$ Her (Mathias \& Waelkens 1995, see below). A summary of the main frequencies that seem to be present in the star is presented in Table 4. The associated NRP degree $l$ and/or azimuthal quantum number $m$ found by various methods are also given.

We included in Table 4 the corresponding superperiod (travelling period of bumps through the stellar disk), namely $|m| P$ for the cases in which a value of $m$ could be estimated. It can be seen that with the exception of the $0.78 \mathrm{c} / \mathrm{d}$ frequency, the superperiods agree within $\sim 8 \%$ : $|m| P=0.73 \pm 0.06 \mathrm{~d}$ (the agreement is better if one takes into account the correction due to the Coriolis force). The reality of this travelling period of bumps is confirmed by an independent estimation using the acceleration of the "moving bumps", that are the signature of high-order 
Table 4. NRP degree $l$ and azimuthal order $m$ for the main frequencies found in this paper deduced with different methods. The corresponding superperiods are given in the last column

\begin{tabular}{llll}
\hline$\nu(\mathrm{c} / \mathrm{d})$ & FDI & $\Omega=1.5(\mathrm{c} / \mathrm{d})$ & $|m| P(\mathrm{~d})$ \\
\hline 0.78 & $l=1-3$ & $l=1, m=-1$ & 1.28 \\
1.29 & $l=1-3$ & $l=2, m=-1$ & 0.78 \\
1.48 & $l=3-5$ & & \\
1.78 & $l=1-3$ & & \\
3.82 & $l=1-3$ & & \\
4.34 & $l=2-4$ & $l=3, m=-3$ & 0.69 \\
4.51 & $l=3-5$ & $l \geq 4, m=-3$ & 0.67 \\
7.80 & $l=6$ & $l \sim 7, m \sim 6^{*}$ & 0.77 \\
\hline
\end{tabular}

*Leister et al. (1994).

NRP modes. They are clearly seen in Fig. 4. In the case of sectoral modes, the superperiod can be written $|m|$ $P=2 \pi V \sin i / a$, where $a$ is the bump acceleration at the line center. Taking $V \sin i=350 \mathrm{~km} \mathrm{~s}^{-1}$, one obtains for the 6 nights $|m| P=0.73 \pm 0.04 \mathrm{~d}$.

\subsection{NRP and rotation in $\eta$ Centauri: A tentative scenario}

It seems clear that $\eta$ Centauri changed its variability pattern somewhere between May 1992 (data secured by SBHB) and May 1993 (LNA photometric data, this paper). The earlier pattern was dominated by a $1.56 \mathrm{c} / \mathrm{d}$ periodicity present in spectroscopic (EW, FWHM, RV) and photometric measurements. In particular, photometric data are well reproduced by a single-wave light curve which corresponds to a $m=1 \mathrm{NRP}$ mode. After the beginning of 1993 and up to mid-March 1995, the star shows a richer frequency spectrum, with four rather intense signals: $1.29,1.78,3.82$ and $4.51 \mathrm{c} / \mathrm{d}$ (see Figs. 2, 3 and Table 4). The changing temporal behavior of the frequency spectrum of multiperiodic Be stars is a subject of current investigation (e.g. Smith 1985; Ružić et al. 1994; Zhiping \& Aying 1997; Śtefl et al. 1997). In the case of NRP, nonlinearity, beating of high amplitude modes and resonant coupling between modes may render rather difficult to establish a definitive picture of the stellar pulsation characteristics. Even if the influence of the observational window function and its associated aliases is properly taken into account, possible intrinsic variability of mode amplitudes may entangle the situation even further. These considerations suggest that many factors can explain the changing variability pattern of $\eta$ Cen.

A remarkable behavior appearing in the nightly FDI analysis of our data can be seen in Fig. 4. For the night of March 15, a mode with $l=2$ is largely dominant. The time resolution is insufficient to allow the corresponding frequency to be determined, but from Table 4 , it can be identified with $1.29 \mathrm{c} / \mathrm{d}$. In the subsequent night (March
16, Fig. 4b) the pattern radically changed, being now dominated by a mode with $l=4$ that can equally be associated with the frequency $4.51 \mathrm{c} / \mathrm{d}$ (see Table 4 ). This sort of alternating condition is also clearly observed for the other March 1995 data, namely that nights 11, 17 show a strong $l=2$ mode, whereas the pattern is dominated by a $l=4$ in nights 12,18 . This $\sim 2$-day mode alternation can be due to a beat modulation linked to the travelling period of bumps/or to the stellar rotation: the repetition of the two distinct NRP dominant surface patterns occurs each $\sim 2$ days $\simeq 3$ superperiods (see above). This is similar to the proposition by SBHB, namely that $1.56 \mathrm{c} / \mathrm{d}$ could be the stellar rotation frequency.

NRP frequencies measured in an inertial frame can be written $\nu=\nu_{0} \pm m\left(1-C_{\mathrm{nl}}\right) \Omega_{\mathrm{rot}}$, where $\nu_{0}$ is the frequency in the stellar frame for a non-rotating star, and $\Omega_{\text {rot }}$ is the stellar rotation frequency. Adopting $\Omega_{\text {rot }}=1.5 \mathrm{c} / \mathrm{d}$ as a working hypothesis and supposing that NRP in $\eta$ Cen are high radial order $g$ modes (in which case $\nu_{0} \approx 0$ and $C_{\mathrm{nl}} \simeq 1 /(l(l+1))$, cf. Dziembowski \& Pamyatnykh 1993; Gautschy \& Saio 1993), some of the frequencies in Table 4 can be reproduced: the corresponding $l, m$ values are reported in the third column of the table. Note that they do not differ significantly from the other results, given the uncertainties. The frequencies in Table 4 are in good agreement with theoretical calculations by Gautschy \& Saio (1993) of unstable NRP $g$-modes in hot B stars (with the exception of the $7.8 \mathrm{c} / \mathrm{d}$ signal). Other high value frequencies $(9.1,10.5,11.5,17 \mathrm{c} / \mathrm{d})$ seem also to be present in the CLEANEST results for the nights analysed separately. They are more compatible with $p$ modes of hot stars, which could exist in the limit between the $\beta$ Cephei and the typical SPB stars in the HR diagram. In these "hybrid" stars, $p$ and $g$ modes would be unstable (Mathias \& Waelkens 1995). Indeed, $\eta$ Centauri could be another example of such intermediate stars: it is a rather luminous B star, located near the lower border of the $\beta$ Cephei region. This subject will be further developed in a forthcoming paper (Janot-Pacheco et al., in preparation).

\section{Summary and conclusions}

In this paper, we analysed new photometric and spectroscopic data of $\eta$ Centauri obtained in 1993 and 1995, observations published by SBHB and Cuypers et al. (1989) and Hipparcos photometric data (1997). A search for periodicities was performed with the help of CLEAN, CLEANEST and the Fourier Doppler Imaging (FDI) methods. Multiperiodic variability seems to be definitively established for this rapidly rotating Be star, and we interpret it in terms of non-radial pulsations. Indeed, the pulsation theory predicts the occurrence of unstable modes in B stars, excited by the metal opacity mechanism.

The star shows amplitude and frequency variability in time scales of years (e.g. before and after early 1993), days 
and hours; they are naturally explained by beating or resonant coupling phenomena.

Night-to-night variations detected during the 8-d observing period are characterized by alternately dominant $l=2$ and $l=4$ modes. This could be due to a beat modulation with the bump superperiod or with the stellar rotation.

The main frequencies found in our analyses (and the associated $l$ values) are: $1.29 \mathrm{c} / \mathrm{d}(l=2), 1.78 \mathrm{c} / \mathrm{d}$ $(l=1-3), 3.82 \mathrm{c} / \mathrm{d}(l=2), 4.34 \mathrm{c} / \mathrm{d}(l=3)$ and $4.51 \mathrm{c} / \mathrm{d}$ $(l=4)$. Those with high amplitude and longer periods can be attributed to high-order $g$-modes, but less intense, short-term ( $\sim$ hours $)$ periodicities have also been detected that can be attributed to $p$-modes. $\eta$ Cen could be thus one of those "hybrid" stars, lying in the HR diagram at the border region between $\beta$ Cep stars ( $p$ pulsators) and the "classical" SPB variables ( $g$ pulsators), showing both $p$ - and $g$ - modes.

Acknowledgements. We thank the referee, Dr. D.R. Gies for judicious comments that significantly improved the paper. The authors thank A.D. de Souza Jr. for helping with photometric data reductions. This research was partially supported by Fapesp (grant No. 95/2719-2), CNRS and CNPq (research grants Nos. 301050/88-RN, 910316/92 and 910180/96-2). S. J. acknowledges financial support by $\mathrm{CNPq}$ and Fapesp through grants No. 301465/95-9.

\section{References}

Cuypers J., 1991, in Proc. ESO Workshop No. 36, Rapid Variability of OB-Stars: Nature and Diagnostic Value, Baade D. (ed.). Garching: ESO, p. 229

Cuypers J., Balona L.A., Marang F., 1989, A\&AS 81, 151

Dziembowski W., 1997, in: Sounding Solar and Stellar Interiors, IAU Symp. No. 181, Schmider F., Provost J. \& Berthomieu G. (eds.). Kluwer, Dordrecht (in press)

Dziembowski W., Pamyatnykh A.A., 1991, A\&A 248, L11

Dziembowski W., Pamyatnykh A.A., 1993, MNRAS 262, 204

Emilio M., 1997, MsC. Thesis, Instituto Astronômico e Geofísico, Universidade de São Paulo

ESA 1997, The Hiparcos and Tycho Catalogues, ESA SP-120
Floquet M., Hubert A.-M., Janot-Pacheco E., Mekkas A., Hubert H., Leister N.V., 1992, A\&A 264, 177

Floquet M., Hubert A.-M., Janot-Pacheco E., Caillet S., Leister N.V., 1996, A\&A 310, 849

Foster G., 1995, AJ 109, 1889

Gautschy A., Saio H., 1993, MNRAS 262, 213

Gies D.R., 1991, in Proc. ESO Workshop No. 36, Rapid Variability of OB-Stars: Nature and Diagnostic Value, Baade D. (ed.). Garching: ESO, p. 229

Gies D.R., 1994, in IAU Symp. No. 162, Pulsation, Rotation and mass Loss in Early-type Stars, Balona L., Henrichs H. and Le Contel J.M. (eds.). Kluwer, Dordrecht, p. 89

Gies D.R., Kullavanijaya A., 1988, ApJ 326, 813

Gray D.F., 1976, The Observation and Analysis of Stellar Photospheres. J. Wiley, New York

Harmanec P., 1988, Bull. Astron. Inst. Czechosl. 39, 329

Hutchings J.B., Nemec J.M., Cassidy J., 1979, PASP 91, 313

Kambe E., Ando H., Hirata R., 1990, PASJ 42, 687

Kennelly E.J., Walker G.A.H., Catala C., et al., 1997 (preprint)

Kennelly E.J., Walker G.A.H., Merryfield W.J., 1992, ApJ 400, L71 (KWM)

Lang K.R., 1992, Astrophysical Data. Planets and Stars. Springer-Verlag, New York

Leister N.V., Janot-Pacheco E., Hubert A.-M., Floquet M., Hubert H., 1994, A\&A 287, 789

Mathias P., Waelkens C., 1995, A\&A 300, 200

Roberts D.H., Lehar J., Dreher J.W., 1987, AJ 93, 968

Ružić Ž., Vujnović V., Pavlovski K., et al., 1994, in IAU Symp. No. 162, Pulsation, Rotation and mass Loss in Early-type Stars, Balona L., Henrichs H. and Le Contel J.M. (eds.). Kluwer, Dordrecht, p. 102

Smith M.A., 1977, ApJ 215, 574

Smith M.A., 1985, ApJ 288, 266

Štefl S., Baade D., Harmanec P., Balona L.A., 1995, A\&A 294, 135 (SBHB)

Štefl S., Baade D., Rivinius Th., Stahl O., Wolff B., Kaufer A., 1997, in A Half Century of Stellar Pulsation Interpretations: A Tribute to Arthur N. Cox, PASP Conf. Series, Bradley P.A. \& Guzik J.A. (eds.) (in press)

Underhill A.B., 1982, in B Stars with and without Emission Lines, Underhill A.B. and Doazan V. (eds.) NASA/CNRS SP-456 monograph, pp. 36-37 and 60

Vogt S.S., Penrod G.D., 1983, ApJ 275, 661

Zhiping L., Aying Z., 1997, PASP 109, 217 